\title{
Investigation of Two-Stage Magnetic Barkhausen Noise Measurement Method at Power Line Frequency
}

\author{
J. PAL'A* AND M. ŠOKA \\ Slovak University of Technology in Bratislava, Institute of Electrical Engineering, \\ Ilkovičova 3, 81219 Bratislava, Slovak Republic
}

(Received July 26, 2015; in final form February 12, 2016)

\begin{abstract}
The aim of the paper was to study the features of measurement of the magnetic Barkhausen noise by the new two-stage method in the high excitation frequency region (power line frequency). The magnetic Barkhausen noise was investigated on grain oriented $\mathrm{Fe}-3 \% \mathrm{Si}$ steel subjected to different heat treatment. By application of an air coil on the output of the power amplifier we were able to linearize the excitation field. At the same time, the air coil suppressed the unwanted noise from the output of the power amplifier. We have found that the two-stage method is able to reveal a peak of the power spectrum even if it is located at low frequency, in contrast to the classical one-stage method. Further, we successfully applied a model of the power spectrum to the real magnetic Barkhausen noise obtained from the two-stage measurement.
\end{abstract}

DOI: 10.12693/APhysPolA.129.383

PACS/topics: 81.40.Rs, 75.60.Ej

\section{Introduction}

The magnetic Barkhausen noise (MBN) exhibits good sensitivity to residual stresses and changes in microstructure of ferromagnetic materials, so the MBN measurement methods are widely used as nondestructive evaluation techniques for inspection of the materials [16]. Measurements of MBN used for assessment of the quality (power losses) of grain oriented $\mathrm{Fe}-3 \% \mathrm{Si}$ steels have already been presented [7-9]. The main potential of the MBN techniques of quality evaluation is that the MBN can be measured directly on the surface of the sheet sample, which allows to monitor rapidly and non-destructively the quality in the transformer sheet production.

The quality of the $\mathrm{Fe}-3 \% \mathrm{Si}$ steels is affected by microstructural features such as grain size, crystallographic texture or inclusions. These features of grain oriented steels are most significantly influenced by a high temperature annealing at the end of the industrial production process. Conventional grain oriented steel is characterized by a sharp Goss texture with huge grain size of few $\mathrm{mm}$ or even cm. It is well known that changes in the microstructural features strongly affect the magnetic domain wall movement and hence the MBN. From the past studies it follows that the investigation of the microstructural changes can lead to inconsistent correlation to the MBN. As an example, the influence of the grain size on the MBN can be taken [7-14]. The situation is complicated by the fact that the MBN is measured in a wide frequency range, from frequencies below $0.1 \mathrm{~Hz}[15,16]$ up to above $100 \mathrm{~Hz}$ [17]. All this could explain why there

*corresponding author; e-mail: jozef.pala@stuba.sk has not been found a clear correlation between the MBN and the quality of the grain oriented steels.

Measurements of MBN in the $\mathrm{Fe}-3 \% \mathrm{Si}$ steel at a low excitation frequency of $1 \mathrm{~Hz}$ and their correlation with the material microstructure were also presented in [18]. Nevertheless, very little has been reported on the MBN at power line frequencies in transformer steels. A higher frequency of $50 \mathrm{~Hz}$ (power line frequency) is not only the working frequency of transformer steels, but measuring at this frequency brings several advantages, such as a higher magnitude of MBN and shorter measurement. On the other hand, at frequencies of tens of $\mathrm{Hz}$ the excitation field interference signal is so large that it complicates the measurement of MBN. In this paper we have investigated the correlation between the MBN measured at power line frequency and the grain size of grain oriented $\mathrm{Fe}-3 \% \mathrm{Si}$ steel samples subjected to different annealing temperatures. The MBN was measured by the two-stage method, which could have better outcomes at higher excitation frequencies [19]. The results were matched with low frequency measurements on the same material presented in [18]. Further, we have studied the influence of the important measuring condition (linearity of the excitation field) on the results at power line frequency, which could help to assess the ability of the MBN method to immediate evaluation of the quality of the steel.

\section{Methods and materials}

A schematic diagram of the system designed for the measurement of MBN is shown in Fig. 1. Besides the twostage method, the system allows measuring the MBN also by the standard one-stage method utilizing a high-pass digital filter. The system uses a U-shape yoke with two driving coils connected in series and wound on the yoke legs made from grain oriented $\mathrm{FeSi}$ sheets. A sinusoidal waveform (with a frequency of $50 \mathrm{~Hz}$ ) from a waveform 
generator is fed to a power amplifier, which amplifies the waveform signal and drives the coils of the yoke. The coil $L$ connected in series with the driving coils is used to linearize the excitation field waveform. The maximum of the excitation field strength was $300 \mathrm{~A} / \mathrm{m}$ at all measurements of MBN.

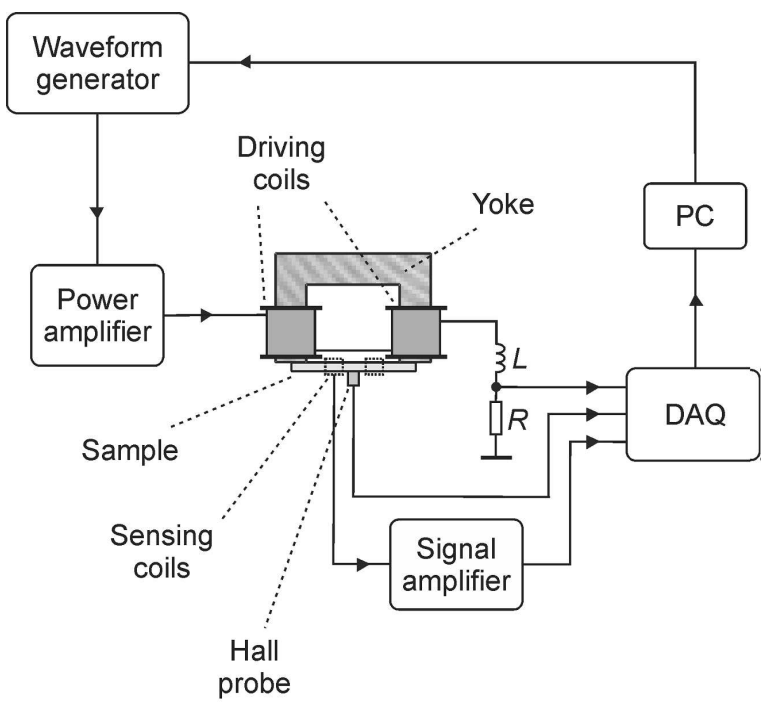

Fig. 1. Measuring setup.

The MBN was acquired using two encircling coils, connected in such a way to have the interference signals generated by exciting yoke cancelled, and placed near the sample center. Both encircling coils had 200 turns and the distance between them was maximum possible to diminish the correlation of MBN signals from sensing coils. The MBN signal from sensing coils was amplified by a preamplifier and then fed to a data acquisition (DAQ) device. Data acquisition was accomplished with the multifunction DAQ device U2542A which features four 16-bit analog to digital input channels. Sampling rate was $500 \mathrm{kHz}$. The personal computer (PC) controlled the waveform generator as well as the DAQ device and collected the measured data.

The DAQ device acquired three signals from a magnetization cycle: the MBN signal induced in sensing coils, the signal from the Hall sensor used for estimating the excitation field, and the voltage on the resistor $R$, proportional to the excitation current. The excitation field interference signal in the two-stage mode was suppressed by using two consecutive waveforms of MBN signal [19]. In this method, we acquired two waveforms of MBN signal from two consecutive magnetization cycles starting from zero magnetic field strength, and then we subtracted them in a computer program. The filtered MBN signal with a duration of one magnetizing cycle was obtained by this method. In the one-stage mode measurement, the MBN signal from sensing coils was acquired only from one magnetization cycle starting from zero magnetic field strength, and it was not additionally filtered. The envelope and power spectrum of MBN signal were then determined. The power spectrum was calculated in a field region around the coercivity, where the sensitivity to microstructural changes is usually largest. These parameters of $\mathrm{MBN}$ were averaged over 30 measurements of MBN signal, which was obtained from one and two magnetizing cycles in the one-stage and two-stage mode measurements, respectively.

To evaluate the waveform quality of the excitation field, the harmonic distortion of the field was calculated as

$$
\mathrm{THD}=\frac{\sqrt{H_{2}^{2}+H_{3}^{2}+H_{4}^{2}+\ldots+H_{n}^{2}}}{H_{1}} 100 \%,
$$

where $H_{n}$ is the root mean square (RMS) value of $n$-th harmonic of the excitation field.

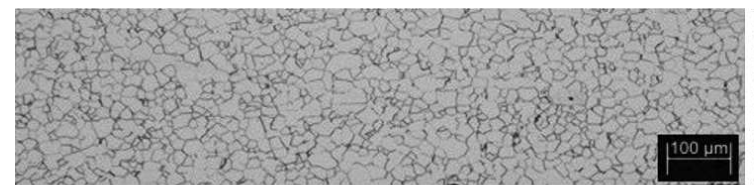

(a)

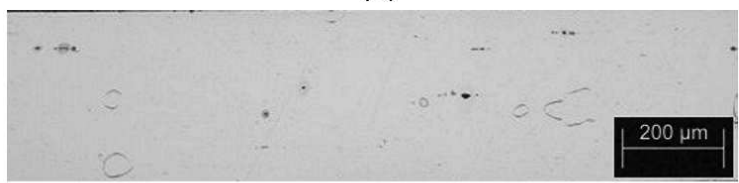

(c)

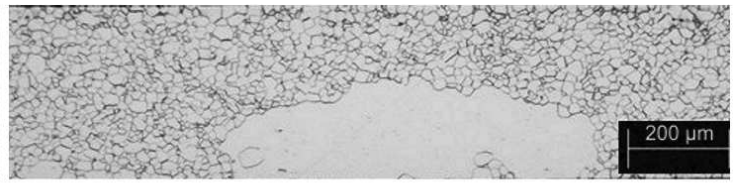

(b)

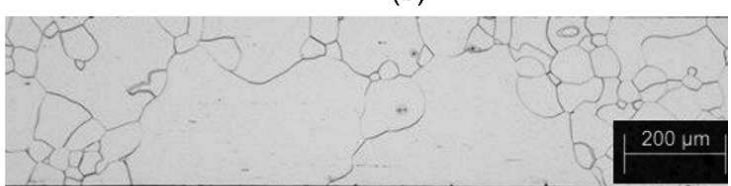

(d)

Fig. 2. Grain structures of samples A-D.

The investigated material was a grain oriented $3 \% \mathrm{Si}$ steel with composition $(\mathrm{C}=0.006, \mathrm{Mn}=0.20, \mathrm{Si}=3.17$, $\mathrm{P}=0.006, \mathrm{~S}=0.008, \mathrm{Cr}=0.029, \mathrm{Ni}=0.031, \mathrm{Cu}=0.46$,
$\mathrm{Al}=0.015, \mathrm{~N}=0.01 \mathrm{wt} \%)$. Samples were cold rolled and cut to dimensions of $40 \times 10 \times 0.3 \mathrm{~mm}^{3}$ with the longer side parallel to the rolling direction. Then the samples 
designated as "A", "B", "C", and "D" were subjected to heat treatment at $850,970,1015$, and $1075^{\circ} \mathrm{C}$, respectively, for $10 \mathrm{~min}$ in pure $\mathrm{H}_{2}$ atmosphere. The evolution of grain size with changing annealing temperature (Fig. 2) was observed in the longitudinal cross-section using microscopy. The microstructure is fully recrystallized after annealing at $850^{\circ} \mathrm{C}$ (see Fig. 2a), when the sample contains small grains with an average size of about $40 \mu \mathrm{m}$. After increasing the temperature to $970^{\circ} \mathrm{C}$ ("B" sample), the abnormal grain growth (AGG) is starting. This leads to bimodal microstructure development, being a mix of small and huge grains of the Goss orientation (see Fig. 2b). The average grain size approaches the millimeter level. The AGG is finished in the "C" sample, containing practically no small grains and having a sharp Goss texture with the average grain size of several $\mathrm{mm}$ (Fig. 2c). Figure 2d reveals that the "D" sample has again bimodal grain structure, similar to that of "B" sample, since at $1075{ }^{\circ} \mathrm{C}$ the selectivity conditions for the AGG are disrupted. The average grain size is between the grain sizes of the "B" and "C" samples.

\section{Results and discussion}

The output impedance of the power amplifier and thus the excitation field waveform can be controlled by the $R-L$ circuit (Fig. 1 ). The MBN is usually measured at a low excitation frequency. The impedance of the driving (or other output $L$ ) coils is negligible in comparison with the impedance of resistor $R$ connected in series with them, and all the output voltage from the power amplifier is across the resistor. Therefore, the excitation field waveform can be assumed to be the same as the output voltage waveform. However, at higher excitation frequencies (power line frequency), the impedance of driving coils increases and cannot be neglected. We have investigated two cases of the influence of the excitation field waveform quality on measurement results. Firstly, we used only the resistor with a negligible resistance, so the entire voltage from the power amplifier was across the driving coils. The resistor was used only for measurement of the distorted excitation current (Fig. 3a).
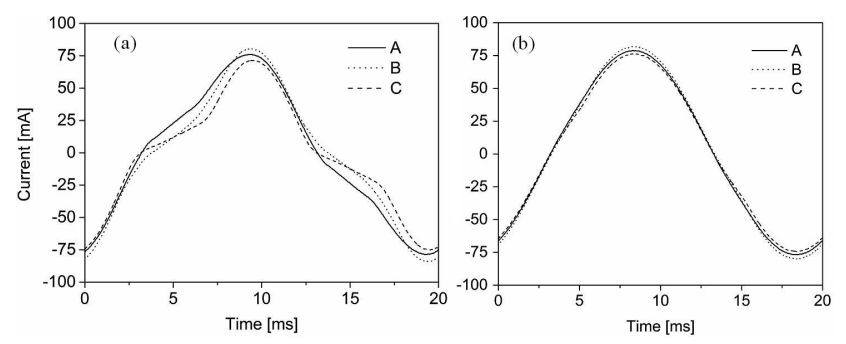

Fig. 3. Excitation current for the "A", "B", and "C" samples measured without (a) and with (b) the output coil.

Consequently, the excitation field (Fig. 4a) is also highly distorted, especially around the coercivity located near the zero field level, which causes a steep decrease of the
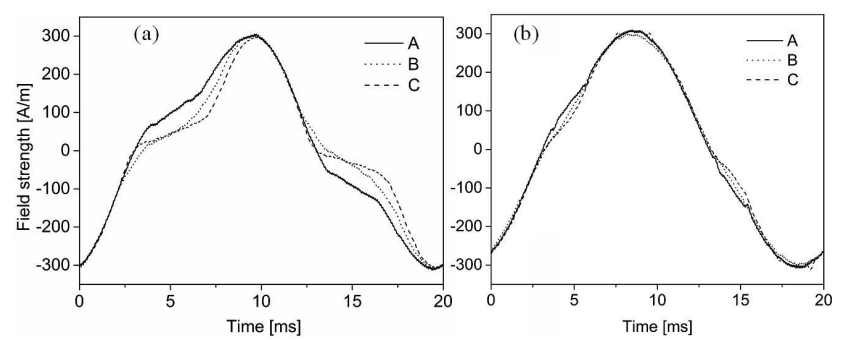

Fig. 4. Excitation field for the "A", "B", and "C" samples measured without (a) and with (b) the output coil.

field slope in this field range (see Fig. 4a). The distortion of the excitation field calculated by Eq. (1) amounts to roughly $25 \%$. The envelopes of MBN for this output impedance (Fig. 5a) show that the dependence of the envelope on the grain size is rather complicated, though it is quite similar to that depicted in [18], where one can find a description of the envelope peaks and their evolution with microstructure changes. Overall, we can say that the "A" sample with the smallest grain size has the highest envelope, which is attributed to the largest number of domain walls in this sample [18]. The other samples, with much higher grain size, have a lower number of domain walls and thus their envelope is smaller.
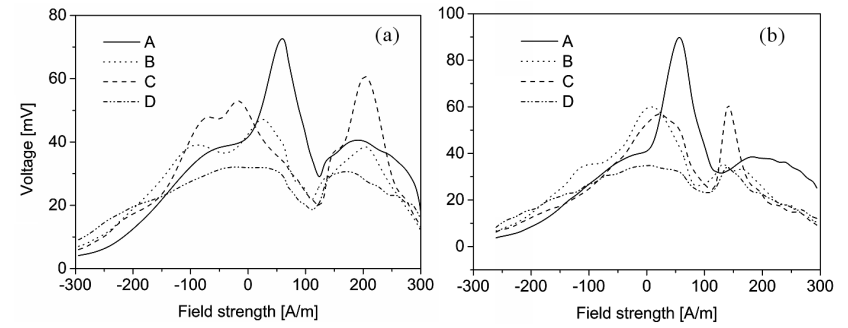

Fig. 5. MBN envelopes from the two-stage measurement without (a) and with (b) the output coil.

Then we added a coreless output coil (air coil in what follows) in series with driving coils, which provides almost frequency independent waveform improvement of the excitation field, if we neglect the resistance of both coils. Such linearization can be achieved also by a resistor with an elevated resistance. However, the output coil has another useful property, as we will see later. The inductance of the coil was chosen so that it was significantly higher than the maximum inductance of the driving coils, which was estimated as about $30 \mathrm{mH}$ by an additional measurement of the driving coils current and voltage. Accordingly, we used an output coil with an inductance of $150 \mathrm{mH}$.

Using the output coil we achieved a linearization of the excitation current and field, as can be seen in Figs. 3b, $4 \mathrm{~b}$. The linearization coil has a similar effect as using a series resistor or a constant current amplifier. After connecting the coil, the increase of the field slope around the coercivity is evident (compare Fig. 4a and b), even though the field is not strictly proportional to the excitation current, as the yoke-sample system is not an ideal closed magnetic 
circuit. More effective linearization can be achieved by using a complex digital feedback. The distortion of the field (THD) decreased to roughly $6 \%$. Nevertheless, the envelopes were only slightly different than in the case of missing output coil (Fig. 5b). The main change is the increase of the "B" sample envelope around the zero field. On the other side, the change in the power spectrum of MBN was more meaningful. Experimental results of the power spectrum of MBN (Fig. 6a) showed that the peak of the spectrum, well known from measurements of the power spectrum of MBN, was revealed only in the case of the two-stage method with the output coil present, presumably due to the lower distortion of an excitation field. When we used the output coil, the spectrum from the two-stage measurement at higher frequencies $(>5 \mathrm{kHz})$ was twice higher than that from the one-stage measurement, as was predicted in [19]. Below $4 \mathrm{kHz}$, the onestage mode spectrum starts to increase rapidly due to a large interference signal. This parasitic signal can be suppressed by a high-pass filter, which also diminishes the peak of the real MBN spectrum. The spectrum from the measurement without the output coil was smaller than that with the coil, apparently due to a lower field rate. We can conclude that we were able to get the real shape of the power spectrum of MBN only when using the twostage method, and after linearization of the excitation field waveform.
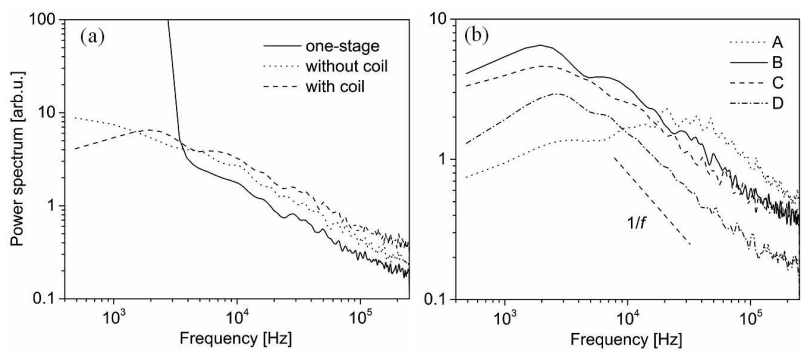

Fig. 6. Typical MBN power spectra, measured for the "B" sample by the one-stage method with the output coil included, as well as by the two-stage method with and without the output coil (a), and all MBN power spectrum curves measured by the two-stage method with the output coil (b).

All experimental power spectra obtained by the twostage method utilizing output coil are shown in Fig. 6b. This figure also contains a $1 / f$ reference line. The spectrum variation with the grain size at higher frequencies fairly correlates with the envelope evolution shown in Fig. 5b. The power spectrum for all samples exhibits one main peak at the frequency $f_{\mathrm{m}}$. Above this frequency, the spectrum decays approximately according to the $1 / f$ law [20]. Durin et al. [21] also observed such a decline, but only within a certain distance from $f_{\mathrm{m}}$. After that point, their spectra scaled with an exponent of $1.5-2$.

The peak of the spectrum moves with the grain size of the samples. For the "A" sample with the lowest grain size, the peak locates around $20 \mathrm{kHz}$. After increasing the grain size, the peak moves towards a smaller frequency of about $2 \mathrm{kHz}$. This shift of the peak frequency with the grain size is in agreement with the model described in [22], where the correlation length parameter $\xi$ of the model, which represents the range of interaction of domain walls with pinning sites, was found to be proportional to the grain size. The dependence of the peak position $f_{\mathrm{m}}$ on the correlation length can be obtained from the ABBM model [23] in the form

$$
f_{\mathrm{m}}=\frac{1}{2 \pi \sqrt{\sigma G \xi /|\mathrm{d} H / \mathrm{d} t|}}=\frac{k}{\sqrt{\xi}},
$$

where $\sigma$ is the electrical conductivity and $G$ is a dimensionless coefficient. As the correlation length $\xi$ is proportional to the grain size, $f_{\mathrm{m}}$ should be inversely proportional to the grain size according to Eq. (2), which is confirmed in Fig. 6b. The grain sizes in the "B", C", and "D" samples are comparable and thus it is difficult to distinguish the peak positions for these samples. The accuracy in the determination of the peak position is generally poor, as was described in [23]. As a consequence, the quantitative assessment of the dependence between the grain size and the peak position is a hard task.

Next, the dependence of the maximum of the spectrum $F_{\mathrm{m}}$ on the correlation length [23] can be written as

$$
\begin{gathered}
F_{\mathrm{m}}=4 S \mu\left|\frac{\mathrm{d} H}{\mathrm{~d} t}\right| \frac{A}{(\sigma G)^{2}} \\
\quad \times\left(\frac{1}{\sigma G S \mu}+\frac{1}{\xi /(S \mu|\mathrm{d} H / \mathrm{d} t|)}\right)^{-2},
\end{gathered}
$$

where $S$ is the sample cross-sectional area, $\mu$ is the permeability and $A$ is the parameter describing the range of spatial fluctuations of short-range domain walls interactions. This suggests that an increase in the grain size should rise the $F_{\mathrm{m}}$, which is in agreement with our experimental results (Fig. 6b). It should be noted that revealing the peak is harder at lower values of $f_{\mathrm{m}}$ due to the closeness of the excitation frequency, as the disturbing noise is large there.

Besides the shift of the peak, a high frequency spectrum of the MBN for the "A" sample also changes in comparison with the other samples. This can be attributed again to the much smaller grain size of the "A" sample, which causes a rise in the number of irreversible processes with short duration. These processes overlap in time [24], which raises the spectrum amplitudes at higher frequencies.

As it was mentioned, the output coil also has the other feature, namely it suppresses an unwanted noise from the output of the power amplifier. This can be regarded as a quite useful feature, as the MBN from the sensing coils is generally a very small signal. As can be seen from Fig. 7, this suppression is quite noticeable. With the coil, the RMS value of unwanted noise decreased about 1.6 times from the noise level measured without the coil at the output of the power amplifier. Better suppression can be achieved by using a higher inductance coil, which is limited by the maximum power amplifier output voltage. 


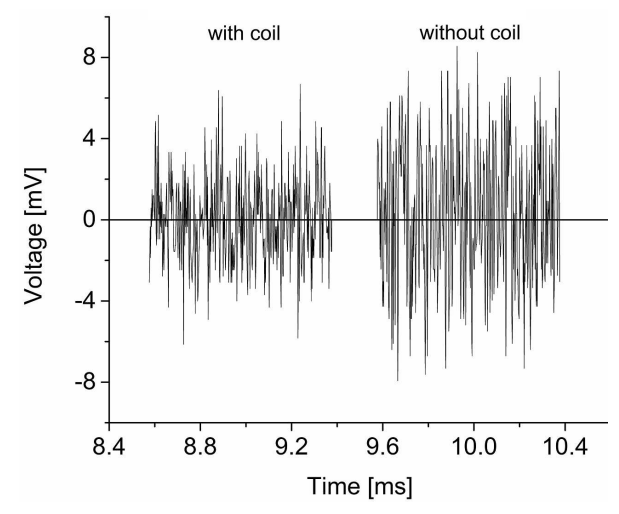

Fig. 7. Disturbing signals measured with and without the output coil.

\section{Conclusions}

A new method of measuring the MBN at power line frequency was tested in this paper. The purpose of this method was to obtain the MBN without using a filter for removing the excitation field interference with the MBN signal. The experimental results proved that the twostage method can help in estimating the true shape of the MBN power spectrum, which was not possible by using the classical one-stage method in our case. We have simultaneously made an improvement of a measuring system, now equipped with the output coil, which increases the signal-to-noise ratio as well as the linearity of the excitation field.

\section{Acknowledgments}

This work was supported in part by the Slovak Research and Development Agency under the contract No. APVV-0062-11, and by VEGA grant No. 1/0571/15.

\section{References}

[1] D.C. Jiles, Czech. J. Phys. 50, 893 (2000).

[2] S. White, T. Krause, L. Clapham, Meas. Sci. Technol. 18, 3501 (2007).
[3] J. Degmová, L. Debarberis, V. Kršjak, Acta Phys. Pol. A 118, 1054 (2010).

[4] J. Pal'a, J. Bydžovský, Acta Phys. Pol. A 126, 855 (2014).

[5] M. Neslušan, D. Blažek, D. Hrabovský, M. Bukovina, Acta Phys. Pol. A 126, 188 (2014).

[6] V. Moorthy, B.A. Shaw, J.T. Evans, NDT $\&$ E Int. 36, 43 (2003).

[7] M. Birsan, J.A. Szpunar, T.W. Krause, D.L. Atherton, IEEE Trans. Magn. 32, 527 (1996).

[8] T.W. Krause, J.A. Szpunar, D.L. Atherton, IEEE Trans. Magn. 39, 562 (2003).

[9] K. Hartmann, A.J. Moses, T. Meydan, J. Magn. Magn. Mater. 254-255, 318 (2003).

[10] R. Ranjan, D.C. Jiles, P. Rastogi, IEEE Trans. Magn. 23, 1869 (1987).

[11] D.-W. Kim, D. Kwon, J. Magn. Magn. Mater. 257, 175 (2003).

[12] S. Yamaura, Y. Furuya, T. Watanabe, Acta Mater. 49, 3019 (2001).

[13] S. Tiitto, IEEE Trans. Magn. 14, 527 (1978).

[14] C. Gatelier-Rothea, J. Chicois, R. Fougeres, P. Fleischmann, Acta Mater. 46, 4873 (1998).

[15] M. Blaow, J.T. Evans, B.A. Shaw, J. Magn. Magn. Mater. 303, 153 (2006).

[16] B. Augustyniak, J. Magn. Magn. Mater. 196-197, 799 (1999).

[17] M. Lindgren, T. Lepistö, NDT $\&$ E Int. 37, 403 (2004).

[18] J. Pal'a, J. Bydžovský, V. Stoyka, F. Kováč, J. Electr. Eng. 59, 58 (2008).

[19] J. Pal'a, J. Bydžovský, J. Magn. Magn. Mater. 361, 88 (2014).

[20] S. Tiitto, S. Saynajakangas, R. Rulka, IEEE Trans. Magn. 12, 406 (1976).

[21] G. Durin, A. Magni, G. Bertotti, J. Magn. Magn. Mater. 160, 299 (1996).

[22] C.C.H. Lo, S.J. Lee, L.C. Kerdus, D.C. Jiles, J. Appl. Phys. 91, 7651 (2002).

[23] B. Alessandro, C. Beatrice, G. Bertotti, A. Montorsi, J. Appl. Phys. 68, 2908 (1990).

[24] D.G. Hwang, H.C. Kim, J. Phys. D Appl. Phys. 21, 1807 (1988). 